\title{
Clinical and Molecular Heterogeneity in Brazilian Patients with Sotos Syndrome
}

\author{
Gustavo H. Vieira ${ }^{a, c}$ Melissa M. Cook ${ }^{a}$ Renata L. Ferreira De Lima ${ }^{d}$ \\ Carlos E. Frigério Domingues ${ }^{c}$ Daniel R. de Carvalho ${ }^{c}$ Isaias Soares de Paiva ${ }^{e}$ \\ Danilo Moretti-Ferreira ${ }^{c}$ Anand K. Srivastava ${ }^{a, b}$ \\ aJ.C. Self Research Institute of Human Genetics, Greenwood Genetic Center, Greenwood, S.C., and \\ ${ }^{b}$ Department of Genetics and Biochemistry, Clemson University, Clemson, S.C., USA; ' Department of Genetics, \\ São Paulo State University, Botucatu, ${ }^{\mathrm{d}}$ Institute of Biology, Federal University of Bahia, Salvador, and \\ e Department of Pediatrics, Faculty of Medical Sciences, State University of Rio de Janeiro, Rio de Janeiro, Brazil
}

\section{Key Words}

Deletion · Mutation · NSD1 · Overgrowth syndrome · PTEN · Sotos syndrome

\begin{abstract}
Sotos syndrome (SoS) is a multiple anomaly, congenital disorder characterized by overgrowth, macrocephaly, distinctive facial features and variable degree of intellectual disability. Haploinsufficiency of the NSD1 gene at 5q35.3, arising from $5 \mathrm{q} 35$ microdeletions, point mutations, and partial gene deletions, accounts for a majority of patients with SoS. Recently, mutations and possible pathogenetic rare CNVs, both affecting a few candidate genes for overgrowth, have been reported in patients with Sotos-like overgrowth features. To estimate the frequency of NSD1 defects in the Brazilian SoS population and possibly reveal other genes implicated in the etiopathogenesis of this syndrome, we collected a cohort of 21 Brazilian patients, who fulfilled the diagnostic criteria for SoS, and analyzed the NSD1 and PTEN genes by means of multiplex ligation-dependent probe amplification and mutational screening analyses. We identified a classical NSD1
\end{abstract}

microdeletion, a novel missense mutation (p.C1593W), and 2 previously reported truncating mutations: $p . R 1984 X$ and p.V1760Gfs*2. In addition, we identified a novel de novo PTEN gene mutation (p.D312Rfs*2) in a patient with a less severe presentation of SoS phenotype, which did not include pre- and postnatal overgrowth. For the first time, our study implies PTEN in the pathogenesis of SoS and further emphasizes the existence of ethno-geographical differences in NSD1 molecular alterations between patients with SoS from Europe/North America (70-93\%) and those from South America (10-19\%).

(c) 2015 S. Karger AG, Basel

Overgrowth syndromes are a heterogeneous group of disorders resulting from the dysfunction of various processes, involving cell proliferation, cell growth or apoptosis [Baujat et al., 2004]. Within this group, conditions such as Sotos syndrome (SoS) (OMIM 117550), Weaver syndrome (OMIM 277590), Beckwith-Wiedeman syndrome (OMIM 130650), Simpson-Golabi-Behmel syndrome (OMIM 312870), and Bannayan-Riley-Ruvalcaba

\section{KARGER 125}

(c) 2015 S. Karger AG, Base

1661-8769/15/0061-0032\$39.50/0

E-Mail karger@karger.com

www.karger.com/msy
Dr. Anand K. Srivastava

J.C. Self Research Institute of Human Genetics Greenwood Genetic Center

113 Gregor Mendel Circle, Greenwood, SC 29646 (USA)

E-Mail anand@ggc.org 
syndrome (OMIM 153480) showed clinical and molecular overlap [Baujat et al., 2004, 2005; Baujat and CormierDaire, 2007].

SoS is an autosomal dominant and probably the most frequent of overgrowth syndromes [Baujat and CormierDaire, 2007]. The syndrome is characterized by childhood overgrowth, advanced bone age, macrocephaly, facial dysmorphic features (elongated facies with a small, pointed chin and prominent forehead), learning disability and speech delay [Cole and Hughes, 1990, 1994]. However, overgrowth and advanced bone age have not to be considered mandatory criteria for a diagnosis of SoS, as demonstrated in a few series of patients [Tatton-Brown et al., 2005; Leventopoulos et al., 2009]. Additional features include skeletal, neurological, renal, and heart defects [Tatton-Brown et al., 2005; Malan et al., 2008]. Patients with SoS also have a tendency for tumorigenesis [Allanson and Cole, 1996]. Haploinsufficiency of the NSD1 gene at $5 \mathrm{q} 35.3$ has been found as the major cause of SoS [Kurotaki et al., 2002]. Point mutations, 5 q35 microdeletions, and partial gene deletions, including one mosaic case, have been detected in $70-93 \%$ of SoS in the UK, France, Germany, and Italy [Kurotaki et al., 2002, 2003; Douglas et al., 2003, 2005a; Rio et al., 2003; Türkmen et al., 2003; Cecconi et al., 2005; Tatton-Brown et al., 2005; Saugier-Veber et al., 2007; Piccione et al., 2011]. Microdeletions involving the complete NSD1 gene are frequently observed in Japanese patients (50 vs. $15 \%$ in non-Japanese patients), whereas intragenic mutations have been found to be more frequent in non-Japanese patients (27-97 vs. 12\% in Japanese patients). Multiplex ligation-dependent probe amplification (MLPA) analysis in 30 Brazilian patients with clinical diagnosis of SoS revealed 3 patients $(10 \%)$ with NSD1 partial gene deletions [Fagali et al., 2009]. Recently, a novel mosaic NSD1 intragenic deletion has been reported in a patient with an atypical presentation of SoS [Castronovo et al., 2013]. Interestingly, several patients with interstitial duplications including NSD1 have been reported with growth retardation, microcephaly and mild-moderate learning difficulties [de Boer et al., 2004; Franco et al., 2010; Dikow et al., 2013; Rosenfeld et al., 2013]. In addition, a de novo 5q35.5 duplication in the immediate vicinity of the NSD1 gene has been identified in a patient with features of SoS which may have resulted in NSD1 haploinsufficiency through a position effect [Kasnauskiene et al., 2011].

With the need to offer a molecular diagnosis to SoS patients who are without NSD1 deletions or alterations, the molecular screening of other candidate genes for overgrowth, such as NSD2, NSD3, NIZP1, RNF135, and
NFIX, in patients with SoS/Sotos-like features has led to the discovery of pathogenetic mutations in RNF135 and NFIX [Cecconi et al., 2005; Douglas et al., 2005b, 2007; Kant et al., 2007; Malan et al., 2010; Priolo et al., 2012; Yoneda et al., 2012]. However, as RNF135 mutations have been associated to a newly defined syndrome, slightly different from SoS [Douglas et al., 2007], to date, only the screening of NFIX has been suggested in SoS patients who lack a molecular diagnosis. In addition, genome-wide SNP array analysis in 26 patients with features of SoS led to the detection of possible pathogenic CNVs, including deletions of 10p12.32p12.31, 14q13.1, Xq21.1q21.31 and a duplication of 15q11.2q13.1 [Visser et al., 2010], thus suggesting that this approach might be complementary to the molecular analyses in SoS patients.

Among other possible overgrowth candidate genes, germ-line mutations in the PTEN gene have been previously associated with a group of disorders called PTEN hamartoma tumor syndrome, which are characterized by macrocephaly, intellectual disability and overgrowth [Yin and Shen, 2008; Hobert and Eng, 2009; Tan et al., 2011]. They include Cowden syndrome [OMIM 158350; Tan et al., 2011], with $85 \%$ of patients carrying a PTEN mutation; Bannayan-Riley-Ruvalcaba syndrome, with $65 \%$ of patients with PTEN mutation, and Proteus syndrome (OMIM 176920), with $20 \%$ of patients with a mutation in the PTEN gene [Zhou et al., 2001, Eng, 2003; Orloff and Eng, 2008]. In addition, PTEN mutations have been found to be associated with autism spectrum disorders and extreme macrocephaly [Butler et al., 2005; Herman et al., 2007], suggesting a possible implication of PTEN in the development not only of overgrowth, but also of cognitive and behavioral functions.

In this study, we have clinically and molecularly characterized a cohort of Brazilian patients with classical SoS clinical features for mutations in NSD1 and PTEN genes. We identified several potential disease causative mutations of the 2 genes: a classical NSD1 microdeletion, a novel NSD1 missense mutation (p.C1593W), 2 previously reported NSD1 truncating mutations (p.R1984X, p.V1760Gfs*2), and a novel de novo PTEN truncating mutation (p.D312Rfs*2).

\section{Materials and Methods}

Patients

Twenty-one Brazilian patients from 20 families were included in this study. These patients were clinically evaluated using the classification described by Cole and Hughes [1994] and MorettiFerreira et al. [1991]. The diagnostic criteria for SoS included typ- 


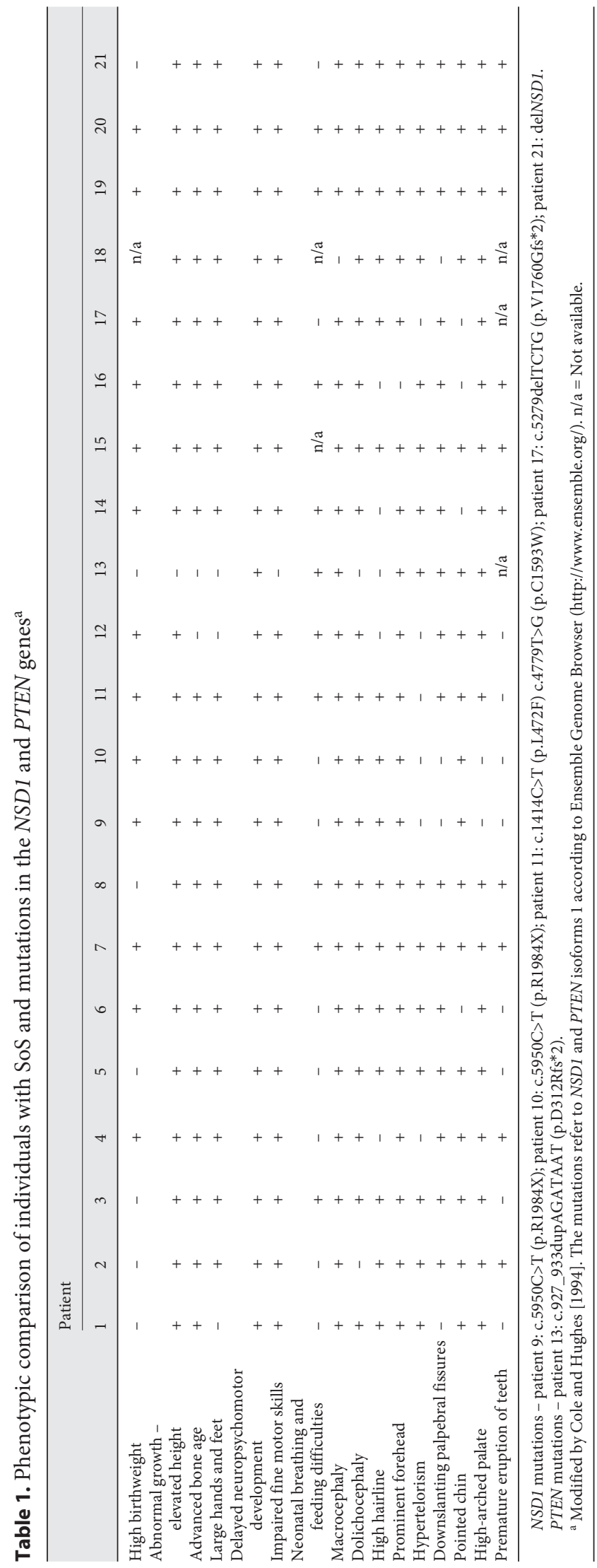

ical facial gestalt in association with at least 1 of the 3 major criteria, namely overgrowth, macrocephaly or developmental delay. The clinical features of all patients are summarized in table 1. Facial features of patients with a classical NSD1 deletion, NSD1 mutations and a PTEN mutation are shown in figure 1. This study was approved by the Research Ethics Committee of Botucatu Medical School, São Paulo State University/UNESP, Brazil (No. OF 536/2004-CEP).

Multiplex Ligation-Dependent Probe Amplification Analysis

MLPA analysis to detect deletions or duplications involving the NSD1 gene region was performed using MLPA SALSA P026-C1_ Sotos panel probe mix kit (MRC-Holland, Amsterdam, The Netherlands). The kit contains one probe for each exon (2 probes for exon 1), 2 probes for the FGFR4 gene residing immediate $5^{\prime}$-end of the NSD1 promoter, 2 probes close to the $5 \mathrm{q}$ telomere and several reference probes on various chromosomes. All reactions were performed following the manufacturer's protocol and instructions. Amplified products were run on 3130xl automated sequencer (Applied Biosystems, Foster City, Calif., USA) and data were analyzed using GeneMarker V1.75 software (SoftGenetics, State College, Pa., USA). The deletion of an exon was determined by a dosage coefficient of 0.5 compared to 1.0 for normal.

\section{Mutation Screening}

The NSD1 and PTEN genes mutation screening was performed by sequence analysis of genomic DNA from 21 patients with SoS features and their unaffected parents, when available. The entire coding region and flanking intronic sequences of both genes (NSD1 exons 2-23 and PTEN exons 1-9) were individually amplified by standard PCR. Amplified products were treated with ExoSAP-IT ${ }^{\circledR}$ (USB Corporation, Affymetrix, Santa Clara, Calif., USA), bidirectionally sequenced by the Sanger method using the BigDye $^{\circledR}$ Terminator v3.1 Cycle Sequencing Kit (Applied Biosystems) and separated on an ABI 3730xl DNA analyzer (Applied Biosystems). Where required, amplicons were subcloned and individual subclones were sequenced to determine the sequence of the normal wild type and the deleted or duplicated alleles in patients. Primer sequences are shown in online supplementary tables 1,2 (see www.karger.com/doi/10.1159/000370169 for all online suppl. material) Sequences were analyzed with DNASTAR SeqMan II software (DNASTAR Inc. Madison, Wis., USA).

\section{Results}

We analyzed a cohort of 21 Brazilian overgrowth patients with a clinical diagnosis of SoS (fig. 1; table 1). MLPA analysis revealed a deletion involving the entire NSD1 gene in patient 21 (fig. 1A; table 1). Analysis of additional probes further revealed that the deletion also included the adjoining FGFR4 gene, located at the $5^{\prime}$ end of the NSD1 gene. The NSD1 gene deletion in this patient was also confirmed by quantitative PCR analysis using a set of primers for NSD1 exon 6.

All patients were subjected to the NSD1 gene mutation screening. Using direct sequencing of the coding region 


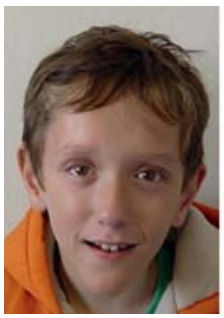

A

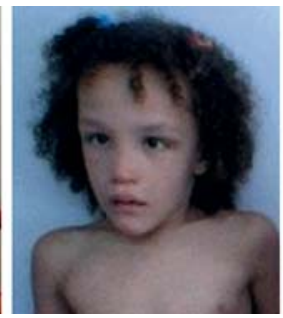

B

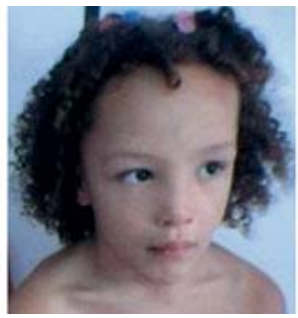

C

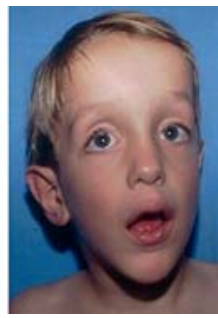

D

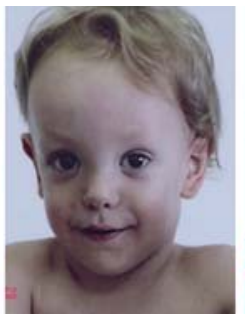

E

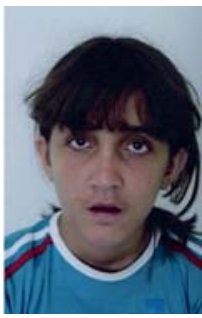

$\mathbf{F}$

Fig. 1. A-E Facial features of patients with defects in the NSD1 gene, including a full gene deletion (A, patient 21), 3 truncating mutations (B, C, twin-sister patients 9, 10 and $\mathbf{E}$, patient 17), and a novel missense mutation (D, patient 11). F Patient 13 carrying a novel de novo truncating mutation in the PTEN gene.

of the NSD1 gene, we identified 4 heterozygous mutations (figs. 2A, 3A): 2 have already been reported in SoS patients, 1 is a novel missense mutation, and 1 is likely a benign variation (table 1 ). In detail, the c.5950C $>\mathrm{T}$ nucleotide alteration in exon 19 was identified in twin sisters, patients 9 and 10 (figs. 1B, C, 2A; table 1). The alteration is predicted to cause an amino acid change from an arginine residue in the NSD1 SET domain to a premature truncation codon at position 1984, p.R1984X (fig. 3A). In patient 11 (fig. 1D; table 1), we identified a novel c. $4779 \mathrm{~T}>\mathrm{G}$ alteration in exon 13 that is predicted to cause an amino acid change from cysteine to tryptophan at residue 1593 (p.C1593W) located between 2 PHD domains (figs. 2A, 3A). This patient also has a novel c.1414C $>\mathrm{T}$ (p.L472F) variation in exon 5 (fig. 2A). Bioinformatic analyses using Polyphen, SIFT, Mutation taster, and PMUT indicated only the variation p.C1593W to be pathogenic, disease causing or not tolerated, and the alteration p.L472F is likely to be benign and polymorphic in nature. In patient 17 (fig. 1E; table 1), a 4-nucleotide deletion (c.5279delTCTG) in exon 15 was identified (fig. 2A). The deletion is predicted to cause a frameshift with Valine at residue 1760 as the first affected amino acid (p.V1760Gfs*2) (fig. 3A). All 3 mutations were absent in 81 normal Hispanic control samples. These variants are not reported in the 1000 Genome Project variation data (http://www.1000genomes.org/data) or dbSNP (http:// www.ncbi.nlm.nih.gov/SNP).

We also screened the PTEN gene in all 21 patients and identified 1 patient, patient 13, (fig. 1F; table 1) with a novel heterozygous PTEN intragenic duplication insertion of 7 nucleotides (c.927_933dupAGATAAT) in exon 8 of the gene which was not identified in the healthy parents (fig. 2B). The duplication insertion creates a frameshift from codon 312 of the 403-amino acid protein, with premature termination at codon 313 (p.D312Rfs*2) (fig. 3B), and thus, this mutation is considered deleterious. The mutation is not reported in the 1000 Genome Project variation data or dbSNP.

\section{Discussion}

All the recruited patients in this study fulfill the SoS diagnostic criteria. Indeed, all the patients showed overgrowth, macrocephaly, intellectual disability, and the typical facial gestalt, except for patient 13, who presented with a less severe SoS phenotype, which did not include pre- and postnatal overgrowth. Of 21 patients, the NSD1 classical deletion was identified in 1 patient, heterozygous NSD1 mutations including a novel missense mutation were found in 4 patients, and 1 patient had a heterozygous duplication causing a novel frameshift mutation in the PTEN gene (table 1).

NSD1 defects reported here, including the entire gene deletion and 2 of the mutations identified in this report (p.R1984X; p.V1760Gfs*2), have been previously reported in patients with SoS, who specifically presented heart anomalies [Cecconi et al., 2005; Tatton-Brown et al., 2005]. In agreement with previous data, patients 9,10 and 17 of the present cohort showed a classical SoS phenotype. However, due to limited available information, we are not able to confirm the association of these mutations and heart malformations in our patients. Similarly, a full SoS clinical picture has been identified in patient 11 , who carried 2 novel missense mutations. However, the bioinformatic analyses suggest that only the p.C1593W alteration is likely to be deleterious and pathogenic.

It is critical to note the detection of NSD1 molecular defects in our cohort of Brazilian SoS patients, namely $19 \%$, and $10 \%$ in a study by Fagali et al. [2009] is very low compared with findings in the European/American co- 
Fig. 2. A Automated sequence chromatograms showing the NSD1 gene mutations in patients 9-11, and 17 . Triplet codons and deduced amino acid residues are shown below the sequence chromatograms. Mutations are indicated by red letters. In patient 17 , we used a sequence chromatogram to deduce the deleted 4 nucleotides. B Automated sequence chromatograms showing the de novo PTEN gene mutation in patient 13. Asterisks indicate a duplication insertion of 7 nucleotides. Amplified products were subcloned, and the sequence of individual clones, with the wild-type and the duplicated alleles in the patient are shown.
Patients 9-10

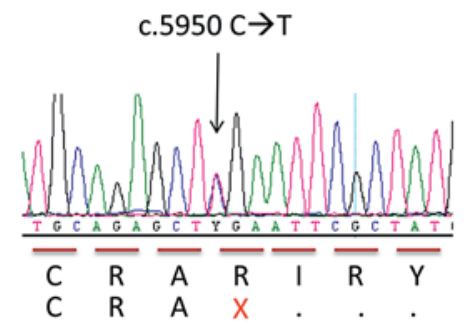

Patient 11

c. $1414 \mathrm{C} \rightarrow \mathrm{T}$

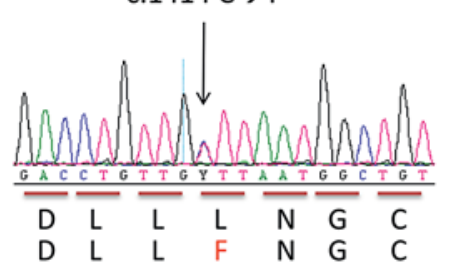

Patient 11

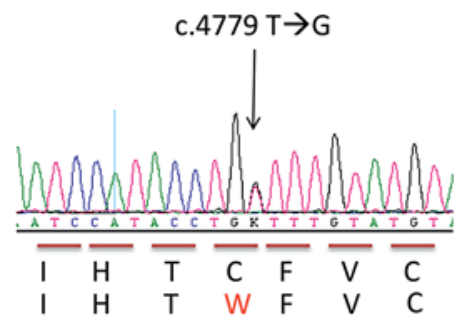

Patient 17

\section{c. 5279 delTCTG}

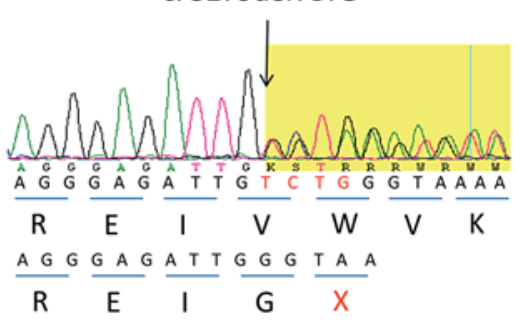

A

Mother
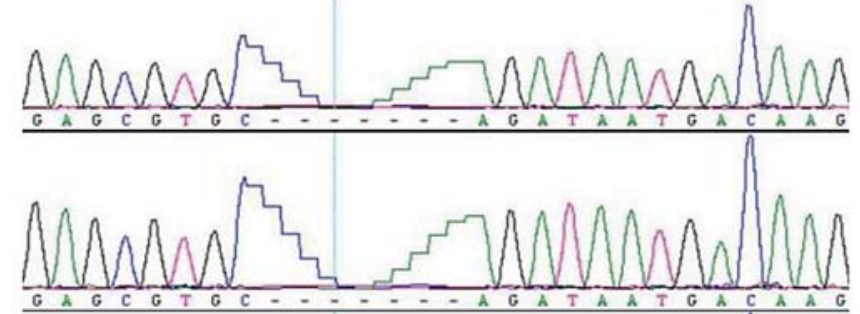

Father

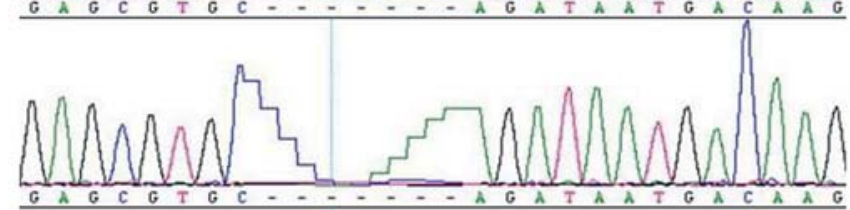

Patient 13

Wild-type allele

(clone)

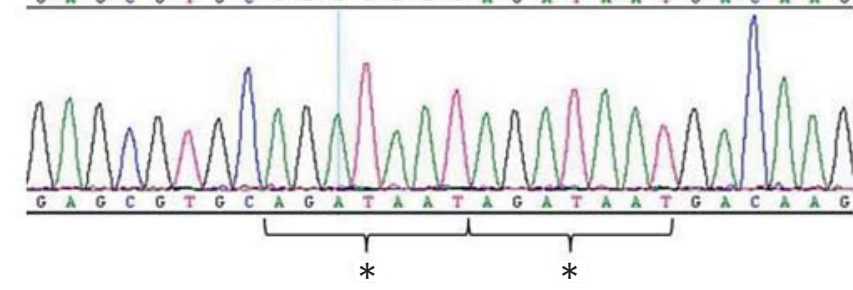

Patient 13

Duplicated allele

(clone) horts (70-93\%). Only the Japanese population, to date, shows such a low rate of mutations but with an elevated rate of NSD1 deletions. Although the number of patients included in our study $(\mathrm{n}=21)$ and in the study reported by Fagali et al. [2009] $(\mathrm{n}=30)$ is too small, it is likely that ethno-geographical differences in NSD1 alterations may exist when patients with SoS from Europe and North America are compared to South American patients. The finding of a duplication located downstream from the NSD1 gene in a patient with classical SoS features [Kas- 
Fig. 3. A Schematic representation of the domains contained in the NSD1 and B PTEN proteins illustrating mutations identified in patients with SoS features.

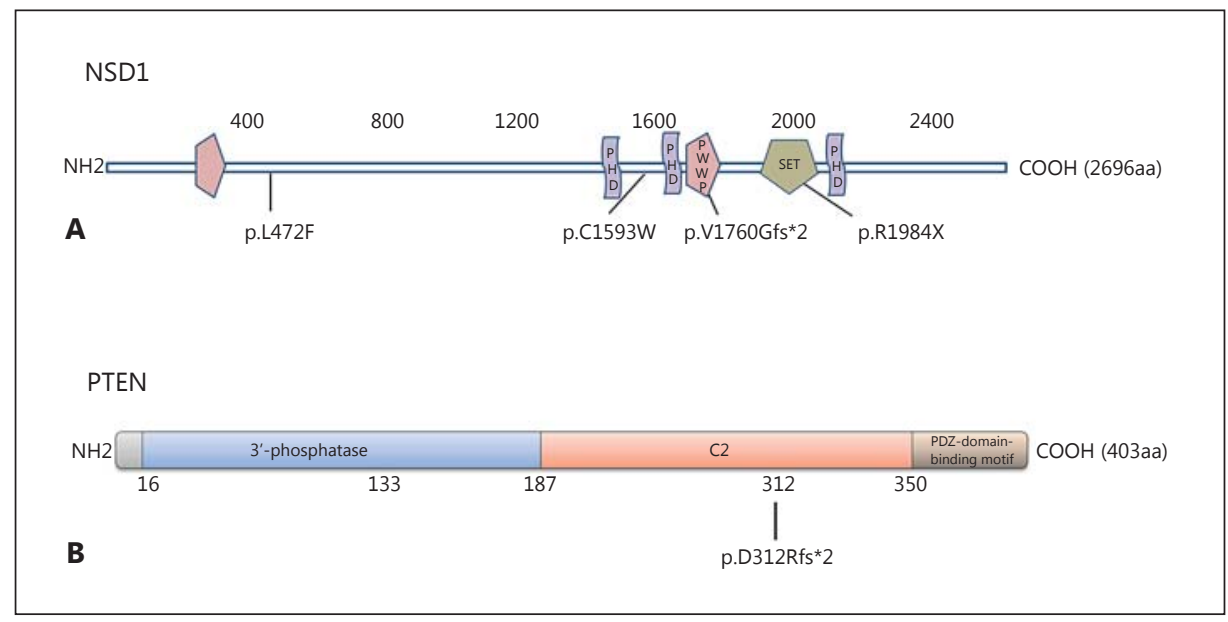

nauskiene et al., 2011] further suggests that the NSD1 gene screening should be extended beyond the coding region to capture all Sotos-associated variants.

As evident, genetic heterogeneity is not an uncommon observation in patients with SoS. In addition, the large clinical overlap among overgrowth syndromes makes it difficult to give a precise diagnosis to several patients and to suggest which genes should be analyzed. Although a majority of patients in our study had classical features of SoS, we identified a deletion and different mutations of the NSD1 gene in only 5 patients, suggesting a likely involvement of other genes in the molecular etiology of patients with features of SoS. Indeed, one of our patients (patient 13) had a novel frameshift mutation in the PTEN gene, previously shown to be responsible for tumor/overgrowth disorders, and a previous report of the NFIX gene mutation in 3 patients with Sotos-like overgrowth features further strengthens the notion that the screening of other genes is critical in patients lacking a defect in the NSD1 gene.

Interestingly, patient 13 (table 1), a thirteen-year-old female of consanguineous parents with an apparent clinical diagnosis of SoS, is the only patient in the present cohort who presents with a less severe SoS phenotype that does not include any sign of overgrowth except for macrocephaly. Conversely, the presence of some features of overgrowth, including macrocephaly, is well documented in patients with PTEN mutations, and thus, the finding of a novel PTEN mutation rather than a NSD1 mutation in this patient suggests phenotypic assessment is a good, but not the best marker of the most likely causative gene. However, in this patient, there were no apparent features indicative of cancer or of mucocutaneous lesions that oc- cur in a high prevalence among patients with Cowden syndrome [Eng, 1997; Pilarski, 2009]. PTEN may play a significant role in a number of molecular pathways regulating cellular proliferation, migration and apoptosis - all processes that are important in the regulation of normal cellular growth [Eng, 1997]. Whereas the PTEN gene is involved in many important biological processes, mutations and polymorphisms in this gene should be investigated in patients with disorders of cell growth, like cancer, and also in patients where overgrowth features are linked to intellectual disabilities, where growth and maturation of the central nervous system may be affected.

While identification of the causative gene and a definitive diagnosis in children with overgrowth is important, some syndromes with this characteristic can increase the risk for cancer as well. Bearing this fact in mind, better recurrence risk counseling can be offered to the families [Tamguney and Stokoe, 2007].

Our findings further emphasize the genetic heterogeneity associated with SoS and expand a role for the PTEN gene in overgrowth/intellectual disability syndromes.

\section{Acknowledgements}

We are grateful to the patients and parents for their participation in this study. We thank Lynn Dukes-Rimsky, Cindy Skinner, and the Molecular Diagnostic Laboratory, Greenwood Genetic Center, for providing assistance at various stages of this study. The study was supported, in part, by a grant from the South Carolina Department of Disabilities and Special Needs, a grant of Fundação de Amparo a Pesquisa do Estado de São Paulo (FAPESP, No. 2005/01880-8) and a grant from the Coordenação de Aperfeiçoamento de Pessoal de Nível Superior (CAPES) foundation, Brazil to G.H.V. 


\section{References}

Allanson JE, Cole TR: Sotos syndrome: evolution of facial phenotype subjective and objective assessment. Am J Med Genet 65:13-20 (1996).

Baujat G, Cormier-Daire V: Sotos syndrome. Orphanet J Rare Dis 2:36 (2007).

- Baujat G, Rio M, Rossignol S, Sanlaville D, Lyonnet S, et al: Paradoxical NSD1 mutations in Beckwith-Wiedemann syndrome and 11p15 anomalies in Sotos syndrome. Am J Hum Genet 74:715-720 (2004).

Baujat G, Rio M, Rossignol S, Sanlaville D, Lyonnet $\mathrm{S}$, et al: Clinical and molecular overlap in overgrowth syndromes. Am J Med Genet C Semin Med Genet 137C:4-11 (2005).

Butler MG, Dasouki MJ, Zhou XP, Talebizadeh Z, Brown M, et al: Subset of individuals with autism spectrum disorders and extreme macrocephaly associated with germline PTEN tumour suppressor gene mutations. J Med Genet 42:318-321 (2005).

- Castronovo C, Rusconi D, Crippa M, Giardino D, Gervasini C, et al: A novel mosaic NSD1 intragenic deletion in a patient with an atypical phenotype. Am J Med Genet A 161A:611-618 (2013).

Cecconi M, Forzano F, Milani D, Cavani S, Baldo C, et al: Mutation analysis of the NSD1 gene in a group of 59 patients with congenital overgrowth. Am J Med Genet A 134:247-253 (2005).

Cole TR, Hughes HE: Sotos syndrome. J Med Genet 27:571-576 (1990).

Cole TR, Hughes HE: Sotos syndrome: a study of the diagnostic criteria and natural history. J Med Genet 31:20-32 (1994).

$\checkmark$ de Boer L, Kant SG, Karperien M, van Beers L, Tjon J, et al: Genotype-phenotype correlation in patients suspected of having Sotos syndrome. Horm Res 62:197-207 (2004).

Dikow N, Maas B, Gaspar H, Kreiss-Nachtsheim M, Engels H, et al: The phenotypic spectrum of duplication 5q35.2-q35.3 encompassing NSD1: is it really a reversed Sotos syndrome? Am J Med Genet A 161A:2158-2166 (2013).

Douglas J, Hanks S, Temple IK, Davies S, Murray $\mathrm{A}$, et al: NSD1 mutations are the major cause of Sotos syndrome and occur in some cases of Weaver syndrome but are rare in other overgrowth phenotypes. Am J Hum Genet 72: 132-143 (2003).

Douglas J, Tatton-Brown K, Coleman K, Guerrero S, Berg J, et al: Partial NSD1 deletions cause $5 \%$ of Sotos syndrome and are readily identifiable by multiplex ligation dependent probe amplification. J Med Genet 42:e56 (2005a).

Douglas J, Coleman K, Tatton-Brown K, Hughes HE, Temple IK, et al: Evaluation of NSD2 and NSD3 in overgrowth syndromes. Eur J Hum Genet 13:150-153 (2005b).

Douglas J, Cilliers D, Coleman K, Tatton-Brown $\mathrm{K}$, Barker K, et al: Mutations in RNF135, a gene within the NF1 microdeletion region, cause phenotypic abnormalities including overgrowth. Nat Genet 39:963-965 (2007).
Eng C: Cowden Syndrome. J Genet Couns 6:181191 (1997).

Eng C: PTEN: one gene, many syndromes. Hum Mutat 22:183-198 (2003).

Fagali C, Kok F, Nicola P, Kim C, Bertola D, et al: MLPA analysis in 30 Sotos syndrome patients revealed one total NSD1 deletion and two partial deletions not previously reported. Eur J Med Genet 52:333-336 (2009).

Franco LM, de Ravel T, Graham BH, Frenkel SM, Van Driessche J, et al: A syndrome of short stature, microcephaly and speech delay is associated with duplications reciprocal to the common Sotos syndrome deletion. Eur J Hum Genet 18:258-261 (2010).

Herman GE, Butter E, Enrile B, Pastore M, Prior TW, Sommer A: Increasing knowledge of PTEN germline mutations: two additional patients with autism and macrocephaly. Am J Med Genet A 143A:589-593 (2007).

Hobert JA, Eng C: PTEN hamartoma tumor syndrome: an overview. Genet Med 11:687-694 (2009).

Kant SG, Kriek M, Walenkamp MJ, Hansson KB, van Rhijn A, et al: Tall stature and duplication of the insulin-like growth factor I receptor gene. Eur J Med Genet 50:1-10 (2007).

Kasnauskiene J, Cimbalistiene L, Ciuladaite Z, Preiksaitiene E, Kučinskienė ZA, et al: De novo 5 q35.5 duplication with clinical presentation of Sotos syndrome. Am J Med Genet A 155A:2501-2507 (2011).

Kurotaki N, Imaizumi K, Harada N, Masuno M, Kondoh T, et al: Haploinsufficiency of NSD1 causes Sotos syndrome. Nat Genet 30:365366 (2002).

Kurotaki N, Harada N, Shimokawa O, Miyake N, Kawame H, et al: Fifty microdeletions among 112 cases of Sotos syndrome: low copy repeats possibly mediate the common deletion. Hum Mut 22:378-387 (2003).

Leventopoulos G, Kitsiou-Tzeli S, Kritikos K, Psoni S, Mavrou A, et al: A clinical study of Sotos syndrome patients with review of the literature. Pediatr Neurol 40:357-364 (2009).

Malan V, De Blois MC, Prieur M, Perrier-Waill MC, Huguet-Nedjar C, et al: Sotos syndrome caused by a paracentric inversion disrupting the NSD1 gene. Clin Genet 73:89-91 (2008).

-Malan V, Rajan D, Thomas S, Shaw AC, Louis Dit Picard H, et al: Distinct effects of allelic NFIX mutations on nonsense-mediated mRNA decay engender either a Sotos-like or a Marshall-Smith syndrome. Am J Hum Genet 87: 189-198 (2010).

Moretti-Ferreira D, Koiffmann CP, Wajntal A, Diament AJ, De Mendonça BB, et al: Macrosomia, macrocrania and motor disorders in childhood, Sotos syndrome (McKusick 11755): report of 7 cases and review of clinical aspects of 198 reported cases [in Portuguese]. Arq Neuropsiquiatr 49:164-171 (1991).
Orloff MS, Eng C: Genetic and phenotypic heterogeneity in the PTEN hamartoma tumour syndrome. Oncogene 27:5387-5397 (2008).

$\checkmark$ Piccione M, Consiglio V, Di Fiore A, Grasso M, Cecconi M, et al: Deletion of NSD1 exon 14 in Sotos syndrome: first description. J Genet 90: 119-123 (2011).

Pilarski R: Cowden syndrome: a critical review of the clinical literature. J Genet Couns 18:13-27 (2009).

Priolo M, Grosso E, Mammì C, Labate C, Naretto VG, et al: A peculiar mutation in the DNAbinding/dimerization domain of NFIX causes Sotos-like overgrowth syndrome: a new case. Gene 511:103-105 (2012).

Rio M, Clech L, Amiel J, Faivre L, Lyonnet S, et al: Spectrum of NSD1 mutations in Sotos and Weaver syndromes. J Med Genet 40:436-440 (2003).

- Rosenfeld JA, Kim KH, Angle B, Troxell R, Gorski JL, et al: Further evidence of contrasting phenotypes caused by reciprocal deletions and duplications: duplication of NSD1 causes growth retardation and microcephaly. Mol Syndromol 3:247-254 (2013).

Saugier-Veber P, Bonnet C, Afenjar A, DrouinGarraud V, Coubes C, et al: Heterogeneity of NSD1 alterations in 116 patients with Sotos syndrome. Hum Mut 28:1098-1107 (2007).

Tamguney T, Stokoe D: New insights into PTEN. J Cell Sci 120:4071-4079 (2007).

- Tan MH, Mester J, Peterson C, Yang Y, Chen JL, et al: A clinical scoring system for selection of patients for PTEN mutation testing is proposed on the basis of a prospective study of 3042 probands. Am J Hum Genet 88:42-56 (2011).

Tatton-Brown K, Douglas J, Coleman K, Baujat G, Cole TR, et al: Genotype-phenotype associations in Sotos syndrome: an analysis of 266 individuals with NSD1 aberrations. Am J Hum Genet 77:193-204 (2005).

Türkmen S, Gillessen-Kaesbach G, Meinecke P, Albrecht B, Neumann LM, et al: Mutations in NSD1 are responsible for Sotos syndrome, but are not a frequent finding in other overgrowth phenotypes. Eur J Hum Genet 11:858-865 (2003).

-Visser R, Gijsbers A, Ruivenkamp C, Karperien M, Reeser HM, et al: Genome-wide SNP array analysis in patients with features of Sotos syndrome. Horm Res Paediatr 73:265-274 (2010).

Yin Y, Shen WH: PTEN: a new guardian of the genome. Oncogene 27:5443-5453 (2008)

Yoneda Y, Saitsu H, Touyama M, Makita Y, Miyamoto A, et al: Missense mutations in the DNA-binding/dimerization domain of NFIX cause Sotos-like features. J Hum Genet 57: 207-211 (2012).

Zhou X, Hampel H, Thiele H, Gorlin RJ, Hennekam RC, et al: Association of germline mutation in the PTEN tumour suppressor gene and Proteus and Proteus-like syndromes. Lancet 358:210-211 (2001). 